\title{
Cross-Layer Algorithm Using Hybrid MIMO Scheme for Video Transmission over Wireless Systems
}

\author{
Martin B. Obando, Walter C. Freitas Jr. and Francisco R. P. Cavalcanti
}

\begin{abstract}
The accelerated development of new technologies in the wireless communication systems has strengthened the data transmission services. Consequently, the implementation of algorithms to offer high quality services becomes necessary. Therefore, in this work, we propose a Cross-Layer algorithm which implements cooperation between the application and the physical layers. In the application layer, it considers a video codification, which generates frames of type $I$ and $P$. In the physical layer, a hybrid Multiple Input Multiple Output (MIMO) scheme with partial channel knowledge at the transmitter exploits spatial diversity gain. Simulation results show that the proposed algorithm improves the performance of the system providing better video quality perceived by the end user.
\end{abstract}

Keywords - Cross-layer, hybrid MIMO scheme, partial channel knowledge, video transmission.

\section{INTRODUCTION}

The video transmission in wireless communication systems is a big challenge that motivates the implementation of algorithms in order to improve the video quality perceived by the end user.

In wireless communication systems, packet loss occurs usually due to the propagation mechanisms of the wireless channel, such as long and short term fadings, which degrade the quality of the received signal.

In recent years, the transmission of video over wireless communication systems and their integration with the Internet is a subject of major interest. This is employed in the IP Multimedia Subsystem (IMS), which is a system of services for next generation networks, and standardized by the 3rd. Generation Partnership Project (3GPP) [1].

The cooperation between the different layers (i.e. Cross-Layer) that compose the stack of protocols in a wireless communication system is an alternative that can be used to satisfy the demands for new services [2]. In the literature, there are several works that propose this mechanism in order to improve the quality of multimedia services, like video.

In the video processing, the scalability provided by the video coding, as well as the ability of classifying the information with priority, makes it possible to use Unequal Error Protection (UEP) mechanisms in order to improve video data transmission. This is achieved by using the cooperative interaction between the application and the physical layers in [3]-[7].

Wireless Telecom Research Group (GTEL), Federal University of Ceará UFC, Brazil, E-mails: martin,walter,rodrigo@gtel.ufc.br
In this work, we propose a Cross-Layer algorithm which includes both the application and the physical layers, where the hybrid MIMO scheme is used to transmit the two kinds of bit-streams, i.e. more or less important bit-streams of video. The strategy used is an improvement of the hybrid MIMO scheme. If the most important bit-stream is identified to be transmitted, the partial channel knowledge in the transmitter is used to choose the best subchannel. This subchannel is allocated to the Vertical Bell Laboratories Layered Space-Time Architecture (VBLAST) layer of the hybrid G2+1 MIMO scheme. This procedure results in a Bit Error Rate (BER) performance improvement of the overall scheme. The atractiveness of this proposal is that the hybrid MIMO scheme is partially improved, in each intra period, and it can be used in conversational applications which have high delay requirements.

The remainder of this paper is organized as follows: In section II, it is given a basic background of MIMO system and some mechanism used in this work. In section III, an overview of video over wireless systems is presented. Section IV describes the assumed system and channel models. In section $\mathrm{V}$, a proposal of the Cross-Layer algorithm is made. Section VI presents performance results and section VII concludes the paper by summarizing the findings.

\section{BACKGROUND ON MIMO SYSTEMS}

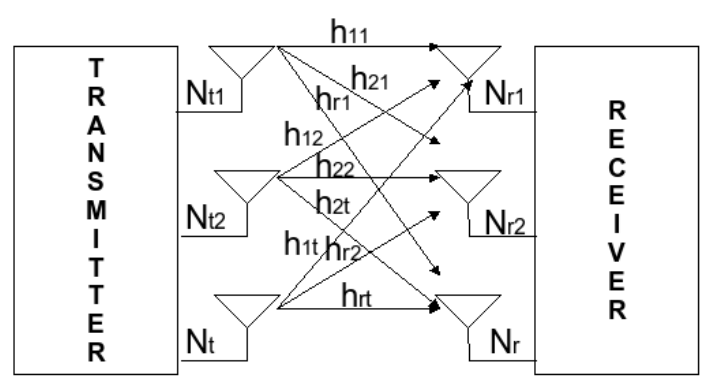

Fig. 1. The MIMO system.

MIMO is a system with multiple antennas for both the transmitter and the receiver. Fig. 1 shows the representation, where $N_{t}$ represents the number of transmitting antennas, $N_{r}$ the number of receiving antennas and $h_{r t}$ represents the gain of each channel between the receiver $r$ and transmitter $t$. This technology can reach high data rates and reliable 
connections, without the need for extra power and bandwidth. The kinds of gains that are achieved with this technology are of multiplexing and diversity. These gains are also achieved, at the same time, with the use of hybrid MIMO schemes, but there is a "trade-off" between the gains [8].

The diversity gain in MIMO schemes is due to the fact that, the reliability of a link can vary widely between the transmitter and the receiver. The use of multiple antennas provides more possibilities to achieve a reliable transmission, since several replicas of the same signal are sent by independent paths. So, there is a high probability that, at least one path is not in deep fading [9].

For multiplexing gain, the bit-stream is divided into smaller bit-streams, which are modulated and transmitted simultaneously and independently by the transmitting antennas. The VBLAST MIMO scheme is an example that exploits this property [10].

In this work, the hybrid MIMO scheme is used with three antennas at the transmitter and receiver. The configuration of the transmitting hybrid MIMO scheme, the partial channel knowledge at the transmitter and the detection algorithm used in the receiver are explained below.

\section{A. Hybrid MIMO Transceiver Structure: $G 2+1$}

This structure consists of three antennas arranged at different parallel layers. The first two antennas form the first layer of the hybrid MIMO scheme. Its configuration corresponds to an Orthogonal Space-Time Block Code (OSTBC) known as Alamounti (G2) [9] and provides diversity gain. The third antenna comprises the second layer and provides multiplexing gain, which is achieved over the time.

The transmission matrix of this scheme is given by:

$$
\mathbf{S}_{G 2+1}\left[T_{1}, T_{2}\right]=\left[\begin{array}{cc}
s_{1} & -s_{2}^{*} \\
s_{2} & s_{1}^{*} \\
s_{3} & s_{4}
\end{array}\right]
$$

in this structure, $K=4$ symbols are transmitted in $T_{s}=2$ consecutive signaling intervals. Therefore, its effective spectral efficiency is given by:

$$
\eta=\left(K / T_{s}\right) \cdot \log _{2}(\mu),
$$

where $\mu$ is considered the cardinality of the modulation scheme. For the G2+1 scheme used in this work, the effective spectral efficiency considering 8-Phase Shift Keying (8PSK) modulation is $6 \mathrm{bps} / \mathrm{Hz}$.

\section{B. Partial channel knowledge at the transmitter}

In the hybrid MIMO scheme, the performance of the multiplexing layers is the limiting part in this scheme, which transmits the symbols without any protection. Antenna selection algorithms can be used to eliminate this problem as in [11].

In [11], the authors propose a scheme to antenna allocation for hybrid MIMO schemes. In this proposal, the best subchannel is estimated at the receiver, based on the power received in each subchannel. This information is sent by the receiver via a return channel. Thus, it is not necessary to send all the channel information, just the subchannels' order.

The partial channel knowledge specified here is considered in the proposed Cross-Layer algorithm to improve the performance, when a more important bit-stream of video is transmitted.

\section{Detection algorithm}

The multiplexing of symbols on the MIMO transmission schemes presents the problem of interference and requires signal processing for their detection.

For the signal reception of VBLAST and of the hybrid MIMO scheme G2+1, it is possible to use linear detection of the symbols by layers. In this process, the linear weighting is done to meet a performance criterion, such as the Minimum Mean Square Error (MMSE) or the Zero Force (ZF) criterion [12].

In the non-linear detection, the technique used is the Successive Interference Cancelation (SIC). In this case, the contribution of the symbols detected successively in the received signal is removed from each layer. Assuming correct decisions with respect to the transmitted symbols, the signal remains free from interference of previously detected symbols, achieving diversity for the next layers and for the subsequent symbols. This algorithm will present a degradation in performance if a previous symbol is incorrectly detected. This is due to its dependent architecture on previously detected symbols. This problem can be alleviated as long as optimal detection ordering of layers is made. When detection ordering is assumed, the first layer to be detected is that with the higher Signal-to-Noise Ratio (SNR). In this situation the SIC approach turns into Ordered Successive Interference Cancelation (OSIC).

In this work, the OSIC detection algorithm was implemented as in [12].

\section{VIDEO OVER WIRELESS COMMUNICATION SYSTEMS OVERVIEW}

The growing demand for multimedia services has made video one of the key parts in the wireless services development.

Each application of video has its own constraints, as presented in Table I [7]. Therefore, strategies are needed in the processing of video, in its compression, transmission and decoding process, to ensure quality for video services, given the limited resources imposed by the wireless communication systems.

The most important video applications considered in wireless communication systems are explained below:

- Download and play: this application performs the compression, transmission and decompression of data separately. The video sequences are compressed and stored on a server before being transmitted. The receiver makes a requisition to download some content of video. Nevertheless, it only plays if a full content of video has been downloaded, free of errors. Multimedia Messaging Service (MMS) is an example of this kind of application. 
TABLE I

SOME CHARACTERISTICS OF TYPICAL WIRELESS VIDEO APPLICATIONS.

\begin{tabular}{|c||c|c|c|}
\hline Video application & Max. delay & CSI Available & Encoding \\
\hline \hline Download-and-play & $\mathrm{n} . \mathrm{a} \cdot$ & - & Offline \\
\hline On-demand streaming & $\geq 1 \mathrm{sec}$ & Partly & Offline \\
\hline Live streaming & $\geq 200 \mathrm{~ms}$ & Partly & Online \\
\hline Multicast & $\geq 1 \mathrm{sec}$ & Limited & Both \\
\hline Broadcast & $\geq 2 \mathrm{sec}$ & No & Both \\
\hline Conferencing & $\leq 250 \mathrm{~ms}$ & Limited & Online \\
\hline Telephony & $\leq 200 \mathrm{~ms}$ & Partly & Online \\
\hline
\end{tabular}

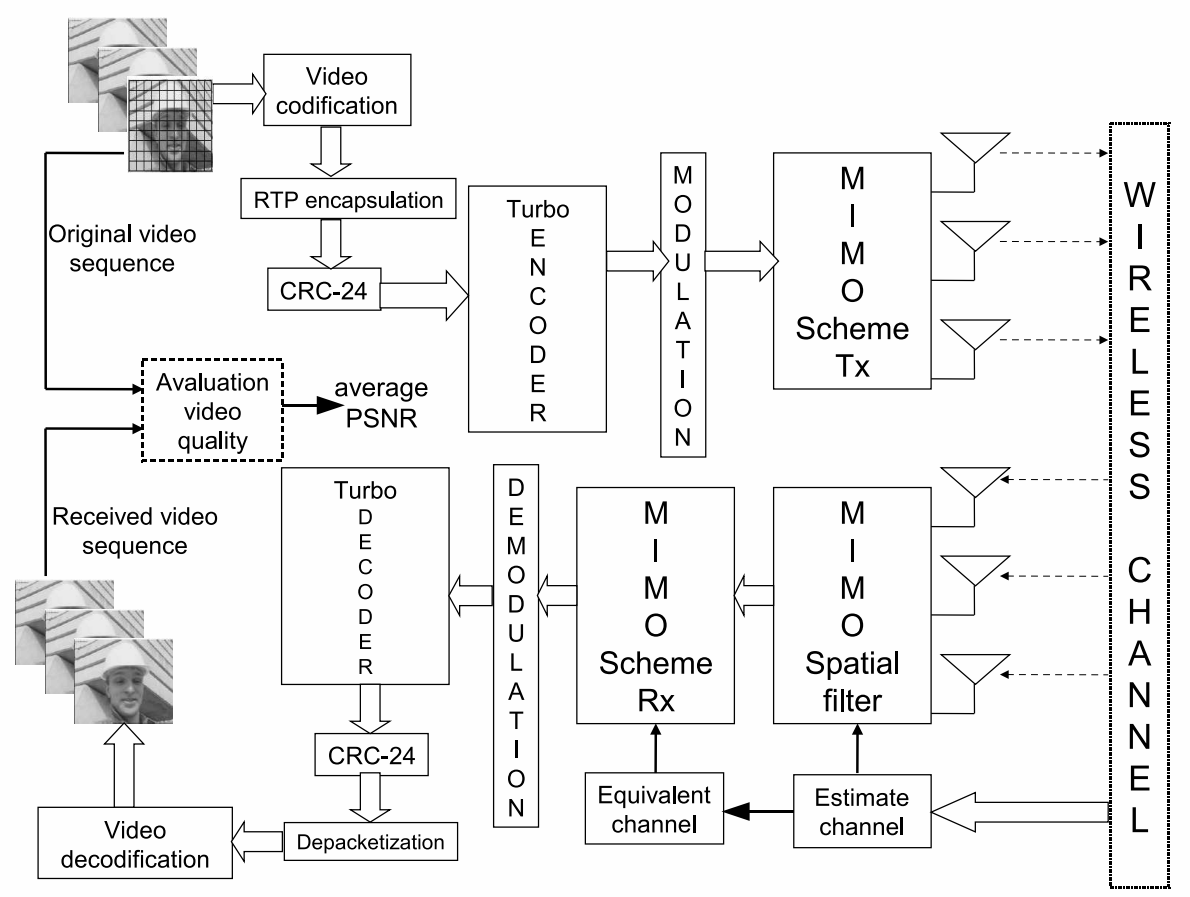

Fig. 2. The simulator system.

- Streaming: in such application, precoded video units are stored on a server, which can be played, during its download, without requiring that all video content be received. In this kind of application, the video on demand is considered.

- Conversational: in its implementation the goal is to minimize the delay inserted in the compression, buffering and decompression process. Therefore, due to its characteristics, it limited the application of compression algorithms and control of errors.

One way to combat the variability of the wireless channel in the video transmission is the use of error control techniques, some of which are included in the video coding standards, and other ones in the systems that provide this kind of services. These techniques can include: the error resilience, error concealment, forward error correction, power and data rate control.

For video conversation in Third Generation (3G) mobile devices, for example, the H.263 and MPEG-4 visual are the most important video coding standards. The H.264 Advance Video Coding (AVC) is also considered, using the baseline profile.

\section{System AND Channel Model}

In this section, we present the implemented framework for video transmission over wireless system and the assumed channel model .

Fig. 2 shows the system implemented for the evaluation of Cross-Layer algorithms that involve the application and the physical layers.

The application layer is composed of the video coding and packetization process. This was set up according to [13] and [14]. The physical layer is composed of the Cyclic Redundancy Check (CRC)-24, the Turbo coder, the modulation and MIMO schemes. Also, an objective method to evaluate the video quality was implemented based on the Peak Signal-to-Noise Ratio (PSNR) [15]. 


\section{A. Channel model}

We consider wireless transceivers equipped with $N_{t}$ transmitting antennas and $N_{r}$ receiving antennas as in [11]. The wireless channel is assumed to have rich-scattering and flat-fading, and signals are assumed to be transmitted through a random channel matrix $\mathbf{H}$ of size $N_{r} \times N_{t}$ whose entries are independent circularly symmetric complex Gaussian random variables. A quasi-static block fading model is assumed where $\mathbf{H}$ is constant during the transmission of $T_{s}$ symbols. In each block we can represent the received signal as in [12]:

$$
\mathbf{Y}=\sqrt{\frac{\rho}{N_{t}}} \mathbf{H S}+\mathbf{N},
$$

where $\mathbf{Y} \in \mathbb{C}^{N_{r} \times T_{s}}$ is the received signal matrix, $\mathbf{S} \in \mathbb{C}^{N_{t} \times T_{s}}$ is the transmitted signal matrix, $\mathbf{H} \in \mathbb{C}^{N_{r} \times N_{t}}$ is the channel matrix, $\rho$ is the average signal to noise ratio, and $\mathbf{N} \in \mathbb{C}^{N_{r} \times T_{s}}$ is the additive noise matrix with i.i.d. complex Gaussian entries, each one with unit variance.

\section{Proposed Cross-Layer Algorithm}

The cooperation between the different layers that compose the stack of protocols in a communication system, Open Systems Interconnection (OSI) [16], allows to build schemes for specific needs in the telecommunication services. In this proposal, the cooperation is realized between the application and the physical layers. Fig. 3 shows one interaction followed by this algorithm.

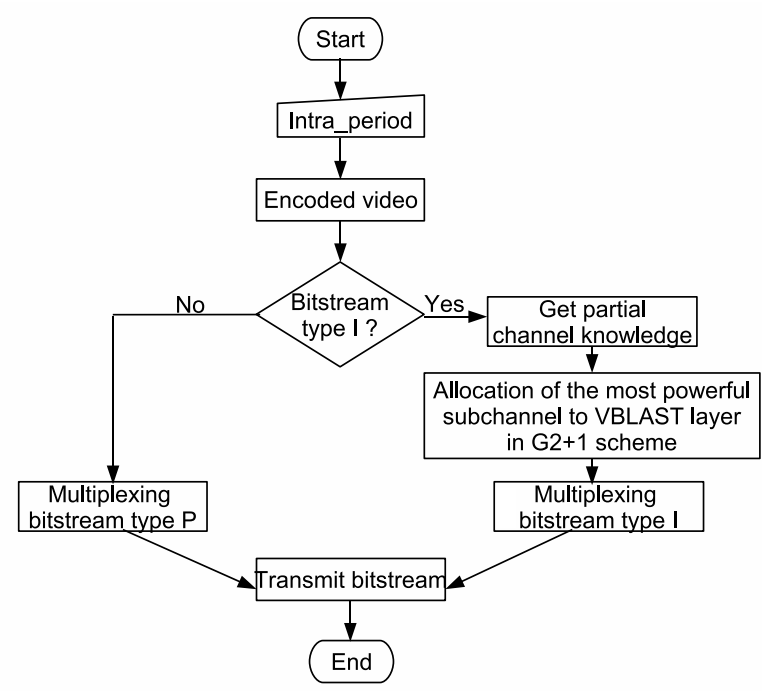

Fig. 3. Flowchart of the Cross-Layer algorithm.

The specification of the Cross-Layer algorithm is as follows: Application Layer

- In the codification of video, frames of type I and P are generated;

- The insertion of frames type I is defined by an intra period;

- In the process of video codification, the type of bit-stream that composes each frame is identified, i.e. bit-stream of type intra-prediction for frame of type I and bit-stream of type Inter-prediction for frames of type $\mathrm{P}$.
Physical Layer

- If the bit-stream is of type inter-prediction, its multiplexing is realized in the default MIMO scheme $(\mathrm{G} 2+1)$;

- If the bit-stream is of type intra-prediction, the partial channel knowledge is required;

- The most powerful subchannel is assigned to the VBLAST layer of the MIMO scheme G2+1;

- The bit-stream of type intra-prediction is multiplexed and transmitted.

In the application layer, during the video codification process, frames of type $\mathrm{I}$ and $\mathrm{P}$ are generated, whereas the insertion of frames of type $I$ is defined by an intra period. This is done, because the frames of type I contain a higher quantity of bits than the frames of type P, i.e. it would need a high data rate of transmission, and its insertion is used to smooth the spreading of errors in case of packet loss.

The packet loss is provoked by the presence of errors inserted during the transmission, due to channel variations in the wireless systems. In the application layer, a smooth strategy is used, which is known as error resilience [1].

In the physical layer, it is used a strategy which consists of UEP to certain types of bit-streams that will be transmitted. For the transmission of the bit-streams it is found that: If the bit-streams correspond to the frame of type P, the MIMO scheme $\mathrm{G} 2+1$ by default is used for its transmission. If the bit-streams to be transmitted correspond to type I frame, then, the partial channel knowledge is required and the powerful subchannel to the VBLAST layer is allocated, which is the unprotected layer in the hybrid MIMO scheme G2+1. Therefore, the performance improves with respect to the BER, as it is shown in [11].

The bit-streams of type I are more protected, because an error free reception is necessary and they can be used for error resilience. The bit-streams that compose the type $\mathrm{P}$ frames did not require high protection, because the type $\mathrm{P}$ frames can be predicted from past type I or P frames correctly received.

\section{Simulation Results}

In this section, the performance of the proposed Cross-Layer algorithm is evaluated by using Monte Carlo simulation. The objective metric used in the evaluation of video quality is the PSNR, which is given by the expression:

$$
P S N R=10 \log _{10} \frac{L^{2}}{M S E},
$$

where $L$ is the dynamic range of allowable image pixel intensities. This metric is function of the Mean Square Error (MSE), and it is expressed in a logarithmic scale [15].

The video coder used was the H.264/AVC baseline profile from [17]. The video encoded bit rate is $64 \mathrm{kbps}$, the frame rate is of $15 \mathrm{fps}$, with intra period of nineteen.

The video sequences used in the simulation were: "Foreman", "Carphone", "Akiyo" and "Coastguard" from [18]. Each video sequence consists of 240 frames, Quarter Common Intermediate Format (QCIF) of size $176 \times 144$ pixels per frame with 4:2:0 sampling. It is commonly used in wireless communication systems. 


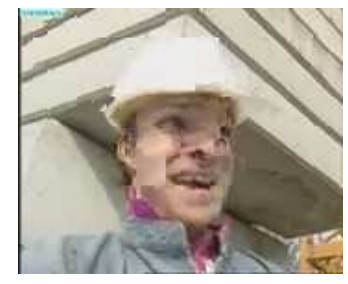

(a) Foreman with CL

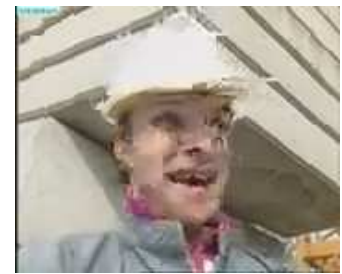

(e) Foreman G2+1

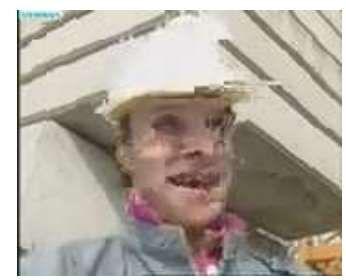

(i) Foreman VBLAST

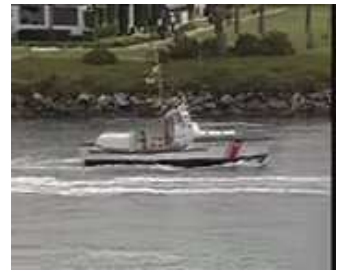

(b) Coastguard with CL

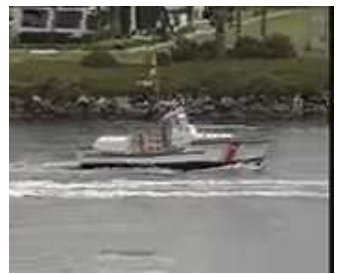

(f) Coastguard G2+1

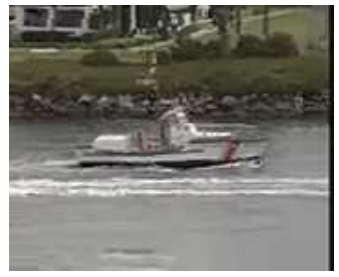

(j) Coastguard VBLAST

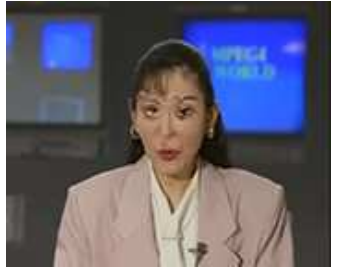

(c) Akiyo with $\mathrm{CL}$

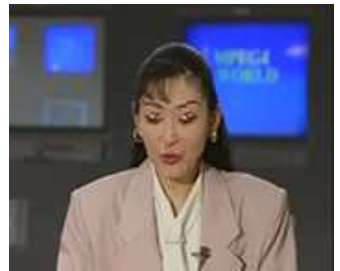

(g) Akiyo G2+1

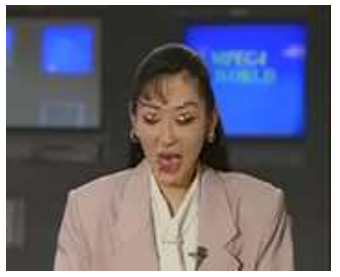

(k) Akiyo VBLAST

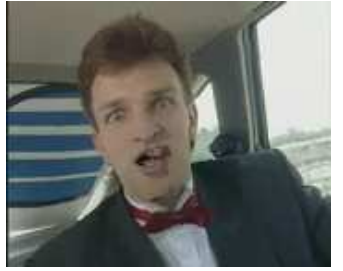

(d) Carphone with CL

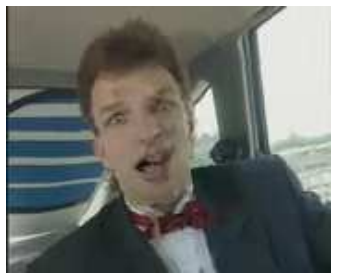

(h) Carphone G2+1

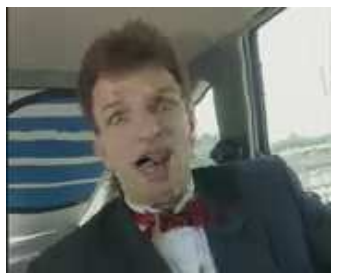

(1) Carphone VBLAST

Fig. 4. Subjective quality of the frame 141 in the video sequences transmitted with $\mathrm{SNR}=11 \mathrm{~dB}$, where frames of the video sequences reconstructed using the algorithm Cross-Layer (CL) appear at the top and the frames with the use of the G $2+1$ and VBLAST scheme are presented below, respectively.

The VBLAST MIMO scheme was implemented in order to evaluate and compare with the performance of the proposed Cross-Layer algorithm. For this purpose, an equal spectral efficiency of the MIMO scheme was considered. The VBLAST scheme with $T_{s}=1$ and a Quadri-Phase Shift Keying (QPSK) constelation. The G2+1 scheme with $T_{s}=2$ and 8PSK constelation. Thus, the spectral efficiency for these schemes is of $6 \mathrm{bps} / \mathrm{Hz}$ with $N_{t}=3$.

In Fig. 4 it is shown, in subjective form, the improvement over the video quality obtained from the use of the Cross-Layer algorithm. The distortion in the frame reflects the effects of packet loss during its transmission. It also reflects the interdependencies that are made during the process of video encoding.

Fig. 5 shows that the proposed Cross-Layer scheme achieves better performance as compared with the VBLAST scheme. When Cross-Layer algorithm is compared with G2+1 MIMO scheme, we can see that there is an improvement in the video quality. This is due to the allocation of the best subchannel over the VBLAST layer of G2+1 MIMO scheme and the reliability enhancement of the bit-stream that composes the frames of type I, which gives refresh or resynchronization to the received video sequence.

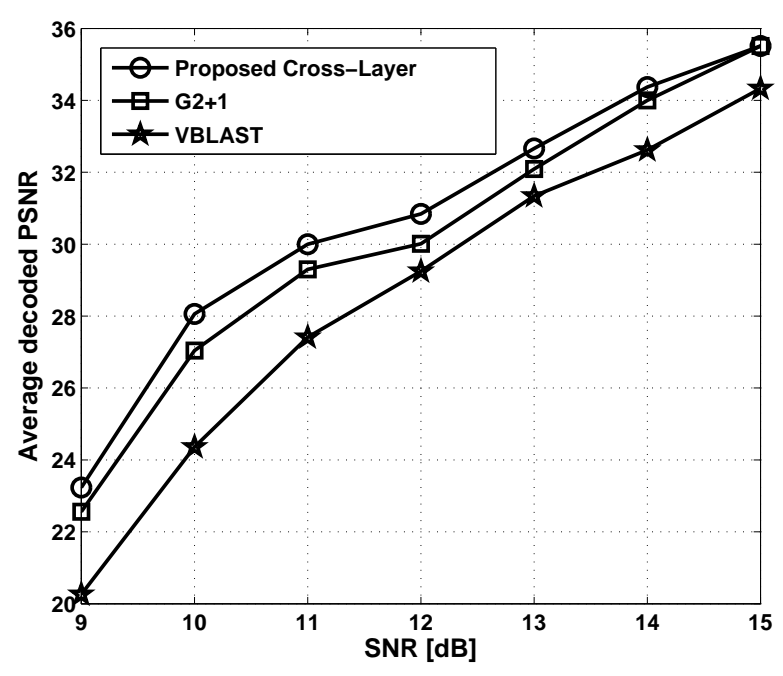

Fig. 5. Performance based on objective evaluation of quality PSNR of the proposed algorithm.

\section{CONCLUSIONS}

In this paper, we propose a Cross-Layer algorithm, which involves the application and the physical layers, where an 
UEP scheme over the bit-streams of video is used during their transmission. The strategy is to improve the performance with respect to the BER of the hybrid MIMO scheme used, when a bit-stream of high importance has to be transmitted. This improvement is achieved in the physical layer by using the partial Channel State Information (CSI) at the transmitter, so that the best subchannel is allocated to the VBLAST layer of the hybrid MIMO scheme. Simulation results show that the objective video quality is improved using this strategy. Therefore, the proposed algorithm is a possible scheme that may be adopted for video transmission over wireless systems. As a perspective, we mention the use of a subjective method for video quality evaluation.

\section{ACKNOWLEDGMENTS}

The author Martin B. Obando is supported by The National Council for Scientific and Technological Development (CNPq).

\section{BIBLIOGRAPHY}

[1] S. Chakraborty, et al., IMS multimedia Telephony over Cellular Systems, John Wiley \& Sons Ltd, 2007.

[2] K.M. El defrawy, et al., "Proposal for a cross-layer coordination framework for next generation wireless systems," IWCMC, Vancouver, British Columbia, Canada, pp. 141-146, July 2006.

[3] H. Zheng, et al., "Space-Time Diversity for Multimedia Delivery over Wireless Channels," IEEE International Symposium on Circuits and Systems, vol. 4, pp. 285-288, May 2000.

[4] C.H. Kuo, et al., "Robust Video Transmission over Wideband Wireless Channel Using Space-Time Coded OFDM Systems," IEEE, vol. 2, pp. 931-936, March 2002.

[5] G. Yang, et al., "Unequal Error Protection for MIMO Systems with Hybrid Structure," IEEE, pp. 682-685, September 2006.

[6] D. Song, et al., "Scalable H.264/AVC Video Transmission Over MIMO Wireless Systems With Adaptive Channel Selection Based on Partial Channel Information," IEEE Circuits and Systems for Video Technology, vol. 17, pp. 1218-1226, September 2007.

[7] T. Stockhammer, "Robust System and Cross-Layer Design for H.264/AVC-Based Wireless Video Applications," EURASIP Journal on Applied Signal Processing, vol. 2006, pp. 1-15, 2006.

[8] L. Zheng, et al., "Diversity and multiplexing: a fundamental trade-off in multiple antennas channels," IEEE Transactions on information theory, vol. 49, pp. 1073-1096, May 2003.

[9] S.M. Alamouti, "A simple transmit diversity technique for wireless communications," Selected Areas in Communications, IEEE Journal, vol. 16 , pp. 1451-1458, October 1998.

[10] G. J. Foschini, "Layered Space-Time Architecture for Wireless Communication in a Fading Environment When Using Multi-Element Antennas," IEEE International Conference on, vol. 1, pp. 436-440, August 2002.

[11] W. C. Freitas, et al., "Hybrid MIMO transceiver scheme with antenna allocation and partial CSI at transmitter side," IEEE-International Symposium on Personal, Indoor and Mobile Radio Communications (PIMRC), pp. 1-5, September 2006.

[12] A. Paulraj, et al., Introduction to Space-Time Wireless Communication, Cambridge University Press, 2003.

[13] H.264/AVC JM Reference Software, "http://iphome.hhi.de/suehring/tml/download/", last access: 21, July 2009.

[14] RFC:3984., "RTP Payload Format for H.264 Video", Network Working Group, February 2005.

[15] Z. Wang, et al., "Mean Squared Error: Love It or Leave It?," IEEE Signal Processing Magazine, vol. 26, pp. 98-117, January 2009.

[16] H. Zimmermannl, "OSI Reference Model-The ISO Model of Architecture for Open Systems Interconnection," IEEE Transactions on Communication, vol. 28, pp. 425-432, April 1980.

[17] A.M. Tourapis, et al., H.264/MPEG-4 AVC Reference Software Manual. Dolby Laboratories Inc., Fraunhofer-Institute HHI, Microsoft Corporation, 2009.
[18] YUV video sequences, "http://trace.eas.asu.edu/yuv/index.html", last access: 21, July 2009. 